\title{
Observer et quantifier le travail pour mieux le comprendre
}

Un dialogue à la rencontre de l'ethnographie et de la statistique

Observing and Quantifying Work To Better Understand It. A Dialogue To

Connect Ethnography and Statistics

Marie Cartier, Anne-Françoise Molinié et Serge Volkoff

\section{(2) OpenEdition}

Journals

Édition électronique

URL : http://journals.openedition.org/travailemploi/9222

DOI : $10.4000 /$ travailemploi.9222

ISSN : 1775-416X

Éditeur

DARES - Ministère du Travail

Édition imprimée

Date de publication : 2 octobre 2019

Pagination : 155-182

ISSN : 0224-4365

\section{Référence électronique}

Marie Cartier, Anne-Françoise Molinié et Serge Volkoff, « Observer et quantifier le travail pour mieux le comprendre », Travail et Emploi [En ligne], 158 | 2019, mis en ligne le 01 novembre 2019, consulté le 07 décembre 2020. URL : http://journals.openedition.org/travailemploi/9222 ; DOI : https://doi.org/

10.4000/travailemploi.9222 


\title{
Observer et quantifier le travail pour mieux le comprendre
}

\section{Un dialogue à la rencontre de l'ethnographie et de la statistique}

\author{
Marie Cartier ${ }^{*}$, Anne-Françoise Molinie ${ }^{* *}$, Serge Volkoff ${ }^{* * *}$
}

\begin{abstract}
Au cours des quatre dernières décennies, les outils permettant d'analyser le travail ont beaucoup évolué. Les données statistiques se sont multipliées, notamment grâce aux enquêtes de la statistique publique, tandis qu'une ethnographie du travail s'est déployée, permettant d'étudier finement les situations de travail. Revue académique publiée avec le soutien de la Direction de l'animation de la recherche, des études et des statistiques (Dares) du ministère du Travail, Travail et Emploi constitue un lieu de rencontre privilégié entre ces deux types de méthodes. Dans cette discussion à trois voix, Marie Cartier, spécialiste de l'ethnographie du travail d'une part, Anne-Françoise Molinié et Serge Volkoff, qui ont participé à la construction des enquêtes statistiques sur le travail d'autre part, échangent autour des possibilités d'articuler ces deux dispositifs méthodologiques et soulignent la manière dont ces deux approches, souvent complémentaires, ont permis de documenter nombre de dynamiques importantes dans l'évolution du travail au cours des quarante dernières années.
\end{abstract}

$\mathrm{D}$ epuis une quarantaine d'années, d'importants changements sont intervenus dans la manière d'analyser le travail : cette période a vu l'émergence de la statistique d'enquête sur le travail en même temps que le déploiement d'une ethnographie visant à rendre compte des situations de travail. Par son positionnement original entre statistique administrative et sciences sociales, Travail et Emploi est l'un des lieux où cette évolution a été particulièrement sensible. À l'occasion de ce numéro spécial célébrant les 40 ans de la revue, nous avons sollicité deux actrices et un acteur ayant

\footnotetext{
* Centre nantais de sociologie (Cens), université de Nantes ; marie.cartier@univ-nantes.fr.

** Conservatoire national des arts et métiers (Cnam), Centre d'études de l'emploi et du travail (CEET); anne. molinie@lecnam.net.

*** CEET; serge.volkoff.visiteur@lecnam.net.
} 
participé à cette histoire afin de comprendre ce qu'elle nous dit du travail. Marie Cartier, professeure de sociologie à l'université de Nantes, a publié en 2010 avec Christelle Avril et Delphine Serre un ouvrage intitulé Enquêter sur le travail. Concepts, méthodes, récits. Anne-Françoise Molinié, démographe, et Serge Volkoff, statisticien et ergonome, tous deux au Centre d'études de l'emploi et du travail (CEET), sont quant à eux spécialistes des statistiques du travail et des conditions de travail et ont notamment coécrit en 2005 un ouvrage sur ce thème : L'Ergonomie et les chiffres de la santé au travail : ressources, tensions et pièges. Enfin, ils ont tous les deux été impliqués dans l'élaboration et la mise en œuvre des premiers dispositifs statistiques nationaux d'analyse des conditions de travail.

Avant de préciser l'apport de ces deux approches, les échanges qu'elles ont noués au fil du temps et les perspectives qu'elles ouvrent, revenons sur leurs définitions respectives.

\section{Observer et quantifier le travail : éléments de définition}

\section{L'ethnographie : observer le travail sans catégorie préconçue}

Marie Cartier. Identifier la spécificité de l'ethnographie du travail est plus compliqué qu'il n'y paraît. Depuis les années 1990, cette approche, qui a connu un renouveau en sociologie en France, se développe au sein de diverses traditions (DODIER, BASZANGER, 1997 ; BEAUD, WEBER, 2012). Par exemple, la place accordée aux paroles sur le travail et à la méthode de l'entretien par rapport à celle de l'observation directe du travail in situ fait débat (ChAPOULIE, 2000), de même que la place accordée à la combinaison des méthodes. Il y a derrière ces questions de méthode des positionnements théoriques différents. La plupart des ethnographes envisagent le travail comme un ensemble d'interactions, mais certains se limitent aux interactions sur le lieu de travail, alors que d'autres s'attachent à réinscrire celles-ci dans des chaînes d'interdépendance et d'interactions à distance, extérieures au lieu et au temps de travail proprement dit. Dans certaines ethnographies, les travailleurs sont dotés de trajectoires sociales et professionnelles et de dispositions différenciées, dans d'autres ils sont d'abord le produit des situations de travail elles-mêmes. D' autres ethnographies encore insistent sur l'interaction entre les travailleurs et le «non-humain » sous toutes ses formes et considèrent le travail comme la combinaison de différentes logiques d'action. L'ethnographe peut sembler transparent ou au contraire très présent : les conditions de son intégration parmi les travailleurs sont alors restituées et analysées, les écarts sociaux entre enquêteur et enquêté dans le rapport au travail étudié pouvant même être utilisés comme leviers de connaissance. De même, certaines ethnographies du travail sont principalement et avant tout ethnographiques, quand d'autres recourent en même temps à l'histoire et aux statistiques, ou bien mobilisent également une enquête qualitative sur un échantillon raisonné de terrains comparatifs. 
Dans l'ouvrage Enquêter sur le travail que j'ai écrit avec Christelle AvRIL et Delphine SERRE et auquel ce que je raconte ici doit évidemment beaucoup, nous avions réservé le terme d' " ethnographie » aux enquêtes qui s'efforcent de saisir, dans la tradition de l'anthropologie, la diversité des scènes sociales sur lesquelles les travailleurs évoluent, la distinguant ainsi de l'enquête par observation participante sur le lieu de travail qui, en France comme aux États-Unis, a été pratiquée dès les débuts de la sociologie du travail. Dans le cadre de la célébration des 40 ans de la revue Travail et Emploi, il est pertinent d'adopter une définition large de l'ethnographie tout en gardant en tête les différentes traditions que je viens d'évoquer. Nombre d'ethnographes du travail sont d'accord avec l'idée qu'ils cherchent à appréhender le travail sans le pré-catégoriser, comme une réalité dynamique et contextualisée, à la fois collective et relationnelle, impliquant des interactions entre une diversité d'humains et de non-humains et qui requiert d'être étudiée le plus directement qu'on peut, sans découpages ni classements préconçus et sans intermédiaire autre que leur présence, leur corps, leur mémoire, leur journal de terrain. Pour des raisons qui tiennent à la fois à la finitude de l'outil d'enquête qui est ici l'ethnographe lui-même et à une épistémologie attentive aux contextes historiques et aux configurations sociospatiales (PASSERON, 1991), l'ethnographie du travail tend vers l'étude d'un cas, vers l'étude d'individus et de groupes circonscrits, et non d'un échantillon représentatif d'une population (SCHWARTZ, 1993 ; BEAUD, WEBER, 2012). La longue durée constitue une dernière spécificité. Alors que l'ethnographie, issue de l'anthropologie, a aujourd'hui gagné en légitimité en sociologie et s'est rapprochée d'autres disciplines comme les sciences politiques et les sciences de gestion, cette contrainte de la longue durée est parfois un peu oubliée.

L'observation directe du travail est une pratique très ancienne. Dans l'article «Atelier» du Dictionnaire du travail (BEvorT et al., 2011), Gwenaëlle Rot cite Jacqueline Frisch-Gauthier mais aussi Émile Zola. Alexandra Bidet, dans l'article "Activité », remonte, elle, au XVIII ' siècle avec l'ingénieur physicien Guillaume Amontons. Mais n'oublions pas que dans l'histoire de la sociologie en France, l'ethnographie a souvent été éclipsée par le traitement statistique d'enquêtes par questionnaire sur échantillon représentatif. En parcourant les titres des 972 articles publiés dans Travail et Emploi entre 1979 et 2018, du numéro 1 au numéro 152, j' ai remarqué que la quantification du travail est présente dès le départ ainsi que l'ergonomie mais que le mot d' « ethnographie » n'arrive, lui, qu' au milieu des années 1990 avec un article de Michel Pialoux (1995, TE $\mathrm{n}^{\circ} 62^{1}$ ) sur les transformations du travail dans l'industrie automobile, puis deux articles de Stéphane BEAUD sur la prise en charge des jeunes non diplômés par les missions locales (BEAUD, 1996a, TE $\mathrm{n}^{\circ} 67$; 1999, $T E \mathrm{n}^{\circ}$ 80). Un peu plus tôt, les recherches sur les processus de connaissance et de reconnaissance des atteintes à la santé liées au travail (ThÉBAUd-Mony, 1992, TE n 54 ; Frigul et al., 1993, $T E \mathrm{n}^{\circ} 56$ ) n'étaient pas tellement éloignées de l'ethnographie tant il s'agissait

1. Les références aux articles parus dans Travail et Emploi sont suivies des initiales de la revue et du numéro dans lequel le texte a été publié. 
de reconstituer l'histoire de vie des travailleurs et de les suivre sur différentes scènes sociales en s'attachant à les relier les unes aux autres dans l'analyse. Dans l'étude du travail et de l'emploi où les sciences économiques sont très présentes et où la quantification fonctionne comme un emblème méthodologique, la légitimité de l'ethnographie ne va pas de soi. C'est même finalement assez extraordinaire qu'une revue comme Travail et Emploi lui ait fait une place en son sein. Ne serait-ce pas une exception française ? J'espère que cela continuera.

\section{Les enquêtes statistiques : comment appréhender les situations de travail par des catégories standardisées}

Anne-Françoise Molinié et Serge Volkoff. Notre appréciation des spécificités de la quantification du travail est évidemment marquée par nos propres pratiques professionnelles, actuelles et passées. Nous avons à l'esprit les particularités des enquêtes qui ont été conduites en France depuis quarante ans, dans le cadre du système statistique public (les enquêtes nationales Conditions de travail, Surveillance médicale des expositions des salariés aux risques professionnels [Sumer], Santé et itinéraire professionnel [SIP], etc.), mais aussi d'enquêtes interprofessionnelles sur de grands échantillons à l'initiative de réseaux de praticiens (Enquête santé, travail et vieillissement [Estev], Santé et vie professionnelle après 50 ans [SVP50], l'observatoire Evrest [Évolutions et relations en santé au travail]), ou encore d'enquêtes locales en entreprises auxquelles nous avons contribué, aux côtés de chercheurs ou praticiens non « quantitativistes».

Les chiffres dont on dispose en France dans le domaine des conditions de travail ou de la santé au travail reposent de fait pour l'essentiel sur des enquêtes directes, et non sur l'exploitation de registres ou de sources administratives, si l'on excepte les décomptes d'accidents du travail et de maladies professionnelles. Ces enquêtes ont été initialement construites en lien avec les interrogations et les pratiques d'acteurs de terrain, et de façon particulièrement étroite avec des ergonomes ou avec des médecins du travail (Gollac, VolKOFF, 2010). Elles sont nées dans une tension entre, d'une part, la crainte des dégâts que pouvait provoquer l'irruption du chiffre dans un domaine complexe et subtil comme les conditions de travail, et, d' autre part, le souhait qu'avaient ces mêmes acteurs de polariser davantage l'attention sur ce sujet, l'absence de chiffre entraînant trop souvent l'invisibilité des domaines concernés (VOLKOFF, MoliniÉ, 1982, $T E \mathrm{n}^{\circ} 11$ ). Cela s'est notamment traduit par le choix de faire une place importante à l'interrogation des travailleurs eux-mêmes, soit directement, soit par l'intermédiaire des médecins du travail ; et par l'attention portée, dans la construction même de l'opération, à ce que la statistique soit un mode de connaissance dont on perçoive clairement les limites, ce qui incite à la combiner avec d'autres approches.

Une deuxième caractéristique, liée à la première, est l'importance accordée à l'explicitation des processus de construction de ces données. Ce travail a revêtu des formes diverses : il s'est mené en prenant appui sur l'analyse des chiffres eux-mêmes, 
notamment lorsqu'ont surgi des résultats surprenants (CÉZARD et al., 1992, TE ${ }^{0} 54$; GollaC, 1997) ; en examinant de près l'activité de travail des médecins enquêteurs et les formes de jugement qui sous-tendent leur activité d' «experts-enquêteurs » (DODIER, 1990, 1993) ; ou en allant réinterroger certains enquêtés pour comprendre comment se construisent et se modifient au cours du temps leurs représentations de leurs conditions de travail (MoliniÉ, 2003 ; GuiHo-BAilly et al., 2009 ; GuIHO-BAIlly, ROQUELAURE, 2013). Ces recherches enrichissent la connaissance non seulement sur le processus de fabrication des enquêtes, mais sur leur objet même, à savoir la façon dont le travail s'inscrit dans des histoires individuelles et collectives. On peut y voir une forme d'antidote aux méfaits potentiels de cette « pré-catégorisation », inhérente aux dispositifs statistiques, sur laquelle Marie insistait à l'instant.

\section{Des différences de regard plus que d'objets : l'informalité et la subjectivité peuvent aussi être mises en statistique}

Anne-Françoise Molinié et Serge Volkoff. Nous ne sommes pas persuadés qu'il y ait des objets pour lesquels la quantification soit spécialement adaptée - et en tout cas, il n'y en a aucun qui lui soit réservé. À l'inverse, il n'y a pas de raison de supposer $a$ priori que tel aspect du travail doive, par nature, être délaissé par les approches statistiques. L'histoire des enquêtes interprofessionnelles sur le travail (GollaC, VOLKOFF, 2010), nationales ou européennes, et le développement des investigations statistiques en entreprises (MOLINIÉ, LEROYER, 2011) montrent que bien des sujets laissés de côté dans un premier temps ont trouvé peu à peu leur place dans les questionnaires. Ce fut le cas tour à tour pour l'usage des outils informatiques, les formes de prescription du travail et d'autonomie, l'articulation entre vie de travail et vie familiale ou sociale, les conflits de valeurs, le rapport avec un public, etc. Sur tous ces thèmes, on a pu se dire d'abord qu'il était peu probant et même risqué de les analyser à l'aide d'explorations chiffrées, mais progressivement des tentatives ont été faites et poursuivies, de façon convaincante à notre avis.

La question serait donc plutôt d'apprécier, sur un objet donné, à quel moment et sous quelles conditions un ensemble de connaissances - qualitatives, sans aucun doute - sur un domaine particulier de la vie de travail paraît assez solide et assez complet pour que la statistique puisse tracer avec soin son propre cheminement au regard de ces connaissances, et développer des articulations avec celles-ci.

Marie Cartier. Je partage votre refus de réserver a priori tel objet à telle méthode. Pendant un temps, j'enseignais aux étudiants que l'ethnographie était particulièrement ajustée pour saisir les pratiques informelles des travailleurs, c'est-à-dire toutes les pratiques qui s'écartent des dimensions officielles du travail. En réalité, comme on l'a rappelé dans Enquêter sur le travail, l'ethnographie peut aussi être mobilisée pour restituer les dimensions officielles du travail dans toute leur complexité. De même, on pourrait être tenté d'affirmer que s'agissant de l'étude des relations professionnelles, l'enquête qualitative par entretiens et monographies d'entreprises comme l'enquête 
documentaire ou statistique seraient plus adaptées tandis que l'ethnographie le serait davantage pour appréhender les relations de travail quotidiennes : entre collègues, avec les chefs et les clients, entre professions, etc. Mais est-ce si sûr ?

Les recherches qui ont mis en évidence la recomposition des conflits au travail en France depuis les années 1990 (imbrication du conflit et de la négociation, essor des formes de protestation individuelle plus ou moins visibles, recours au registre juridique, etc.) à partir d'une exploitation statistique de l'enquête Relations professionnelles et négociations d'entreprise (REPONSE), dont le questionnaire aborde d'ailleurs des pratiques parfois peu formalisées (AMOSSÉ et al., 2008), ont au contraire su tirer parti de la combinaison des méthodes pour ré-interroger la catégorie même de « conflit » et sortir du simple constat de la baisse des grèves. L'ethnographie offre certes un accès privilégié aux significations que les travailleurs donnent aux interactions et aux événements : les refus d'heures supplémentaires sont-ils individuels ou collectifs ? Et sont-ils ou non conflictuels ? Cela peut toutefois aussi se faire par les méthodes statistiques par exemple en croisant, comme le permet l'enquête REPONSE, les perceptions des employeurs et des représentants des salariés quant à l'existence ou la nature de telle ou telle forme de conflit (BÉROud et al., 2008). De même, l'approche ethnographique des trajectoires sociales et professionnelles et du travail concret de représentation des salariés n'est-elle pas précieuse pour saisir les contraintes et ambivalences de la position des permanents syndicaux (MISCHI, 2016) ? Finalement, importer l'ethnographie ou, à l'inverse, la statistique dans un champ de recherche où elle est peu habituelle aide souvent à questionner les représentations conventionnelles du travail et des relations de travail et à renouveler les catégories d'analyse.

\section{Lorsque l'ethnographie se déploie vers les classes dominantes}

Marie Cartier. Je constate toutefois qu'on fait souvent de l'ethnographie quand il est impossible de faire des entretiens ou d'avoir accès à des statistiques. Soit que celles-ci n'existent pas, soit qu'elles soient biaisées. Et si aucun aspect du travail n'est inaccessible à l'enquête statistique, en revanche je pense que le dispositif du questionnaire est socialement sélectif et que l'approche statistique ne saisit sans doute pas avec la même efficacité les points de vue de toutes les catégories de travailleurs. La quantification du travail dont on parle n'est-elle pas aussi très liée à cette configuration sociohistorique que Robert Castel a appelé la « société salariale »? Souhaitant étudier le travail dans les espaces ruraux ukrainiens, Pierre DEFFONTAINES (2018) s'est heurté au délitement de l'encadrement légal et statistique du marché du travail dans ce pays de l'ex-bloc soviétique, qui connaît depuis les années 1990 une instabilité économique permanente. Il n'avait pas d'autre choix que de privilégier l'enquête ethnographique. Celle-ci lui a permis de mettre en évidence la reproduction du pouvoir des grandes entreprises agricoles, qui repose désormais non plus sur le salariat mais sur d'autres formes de relation de travail rémunéré et sur la location des terres dont les travailleurs sont devenus propriétaires. On doit ainsi à l'ethnographie de mieux connaître les 
activités économiques des populations qui, au cœur des riches sociétés occidentales, évoluent en marge de ce qu'on appelle communément le « travail », et qui correspond en fait à l'emploi. Je pense, parmi de nombreux exemples, à l'enquête de Philippe BouRGOIS (2013) sur le commerce de la drogue à New York dans les années 1980, une activité économique qui se révèle tout aussi imprégnée de la croyance économique dans la liberté d'entreprendre et la réussite individuelle que celles qui se déploient dans la légalité. Et à l'enquête récente d'un collectif d'universitaires sur le « travail de subsistance » des classes populaires à Roubaix (COLleCtif RoSA BonHeur, 2017) : les hommes et les femmes que la désindustrialisation et la précarisation de l'emploi rejettent aux marges du marché du travail officiel sont loin d'être aussi inactifs que leur catégorisation administrative ne le laisse supposer. L'ethnographie économique (DUFY, WEBER, 2007) nous aide ainsi à rompre avec la perception à la fois administrative et savante du travail comme salariat né du capitalisme industriel et de la société salariale des Trente Glorieuses. Adaptée à l'étude des pratiques de travail et du rapport au travail dans les marges du salariat et, plus généralement, dans les classes populaires, celles-ci tendant à échapper aux enquêtes par questionnaire, l'ethnographie ne leur est pas pour autant réservée.

C'est au contraire un acquis des développements récents de la recherche en sociologie du travail et des professions que d'avoir étendu l'ethnographie du travail aux professions moyennes et supérieures du commerce et de la finance, professions qui prennent de plus en plus d'importance dans notre économie. Ainsi l'ethnographie du travail des traders (GODECHOT, 2005), des banquiers (MOULÉVRIER, 2010), des agents immobiliers (BERNARD, 2017) ou encore du capital (HERLIN-GIRET, 2019) s'est-elle avérée indispensable pour appréhender les mutations du capitalisme financier. Comme l'ont montré les travaux de Michel Villette (par exemple Villette, Vuillermot, 2005), les pratiques de travail aux marges de la légalité n'étant pas moins présentes dans ces professions dominantes, l'ethnographie est aussi utile pour les aborder. Plus largement, l'ethnographie sait souvent repérer de bons observatoires de la recomposition des croyances économiques et des injonctions managériales : échanges entre conseillers et jeunes chômeurs dans les missions locales (BEAUD, 1996a, TE $n^{0}$ 67), épreuves orales du concours de facteur à la Poste (CARTIER, 2001), formation en « développement personnel » (STEVENS, 2013, TE $\mathrm{n}^{\circ}$ 133), formation associative $\mathrm{au}$ « leadership » (WAINSZTOK, 2017, TE $\mathrm{n}^{\circ}$ 151). À travers ces terrains circonscrits, l'ethnographie du travail a contribué, aux côtés d'autres méthodes et disciplines, à l'objectivation du nouvel esprit du capitalisme (BolTANSKI, ChiaPello, 1999) et à la mise en évidence de sa dissémination, des entreprises aux associations et à la fonction publique d'État. L'ethnographie a aussi permis de montrer que si ces nouvelles valeurs managériales telles la mobilité et l'engagement dans des « projets » sont loin de remettre en cause l'ordre social et sexué ainsi que le prétendent leurs promoteurs, elles n'en ont pas moins des effets sociaux concrets telle la dévalorisation symbolique accrue des travailleurs les moins dotés en ressources scolaires ou encore de l'éthique professionnelle du service public. 


\section{Angles morts, limites et complémentarités des deux approches}

\section{Établir des connaissances par l'analyse de la régularité statistique : la force du nombre est aussi sa faiblesse}

Anne-Françoise Molinié et Serge Volkoff. S'agissant des statistiques du travail, nous ne parlerions pas d'angles morts : on l'a dit, la quantification peut au contraire jouer un rôle dans la mise en visibilité des conditions de travail, de leurs déterminants et de leurs effets, dont certaines composantes tendent à être masquées ou déniées (GolLAC, VOLKOFF, 2006). Certaines limites de l'approche quantitative, en revanche, valent d'être soulignées, car le débat social sur les questions du travail, à l'échelle d'un pays comme d'une entreprise, peine à disposer d'un temps et d'une ampleur d'échanges suffisants, et se contenterait volontiers d'un petit nombre d'indicateurs qui «parlent d'eux-mêmes »: les taux d'accidents et le nombre de maladies professionnelles déjà évoqués, le niveau de l'absentéisme, le volume des heures supplémentaires, la fréquence des réunions des comités d'hygiène, de sécurité et des conditions de travail (CHSCT, tant que ceux-ci ont existé), voire les crédits consacrés à l'amélioration des conditions de travail. Ces informations chiffrées ont leur intérêt mais leur caractère réducteur, trompeur parfois, a été bien montré (ThéBaud-Mony, DaUbas-LetourneuX, 2001, TE n ${ }^{\circ} 88$ ) avec, comme le souligne Marie, une mise à l'écart à peu près systématique du travail informel, mais aussi avec le piège connu de la «circularité » : ces indicateurs acquièrent un statut de but mesurable et, dès lors, des pratiques s'enclenchent, qui visent à influencer ces chiffres eux-mêmes au moins autant que la réalité qu'ils recouvrent.

Au-delà de ces « données » (très « construites » en réalité ; CuRIE, 1995), les enquêtes par questionnaire ont permis d'élargir les champs d'investigation, de diversifier les angles d'attaque d'un même objet, de prendre en compte les points de vue des travailleurs eux-mêmes, avec leur dimension intégratrice d'un ensemble de caractéristiques du travail. Des limites demeurent pourtant... Mais il n'est pas nécessaire de vouloir à tout prix les déplacer ou les franchir ! La principale est due à l'immense variabilité des situations de travail : d'un poste de travail à un autre bien sûr, mais aussi au cours du temps, selon les moments, les incidents, les modifications parfois infimes d'un produit ou d'un équipement, les comportements disparates de clients ou d'usagers, tout ce qui réclame, pour être vraiment apprécié, une forme de « maïeutique » (TEIGER, LAVILLE, 1991, TE n ${ }^{\circ} 47$ ), ouvrant à un travail d'analyse des conditions de travail par celles et ceux qui les vivent et - pour partie - les transforment.

\section{L'ethnographie, de la singularité des situations observées à la critique des ethnocentrismes}

Marie Cartier. N'oublions pas les ethnographes ! Saisir les situations de travail dans toute leur complexité et singularité en nouant des relations interpersonnelles avec celles et ceux qui les vivent, c'est aussi leur métier. On leur reproche encore souvent 
des biais de sélection dans leur population, ou on leur interdit de généraliser leurs résultats sous prétexte que la population étudiée serait trop petite. Mais c'est faire de l'ethnocentrisme statistique, c'est évaluer l'ethnographie à l'aune du raisonnement inférentiel qui n'est pas le sien, puisqu'elle repose sur l'étude approfondie d'un cas qu'elle sait «biaisé » et dont elle s'efforce par conséquent de restituer les conditions de possibilité et l'ensemble des facteurs qui en façonnent la singularité. L'ethnographe monte en généralité en dégageant des «relations causales singulières » et non en déduisant le tout d'une partie. Son but n'est pas de chercher des « régularités à propos d'une population à laquelle il n'accède pas » (GROs, 2017, pp. 130-131) mais de singulariser et contextualiser le plus possible le cas qu'il étudie afin de le relier et de le comparer à d'autres cas. Quand l'ethnographie cherche à expliquer les variations des pratiques de travail, elle ne peut certes pas cerner le poids respectif des facteurs explicatifs de ces variations. En revanche, elle s'avère particulièrement efficace pour cerner ces facteurs dans leur diversité et, surtout, la façon concrète dont ils agissent en se combinant dans un contexte donné (SERRE, 2009).

À partir d'une enquête ethnographique sur les chantiers du bâtiment en région parisienne, Nicolas Jounin (2009) a ainsi montré que la gestion ethnique de la main-d'œuvre s'expliquait non seulement par les stéréotypes ethniques mobilisés par les employeurs pour justifier la division du travail mais aussi par les propriétés juridiques des travailleurs (avoir ou non la nationalité française, être ou non en situation régulière). L'ethnographie permet souvent d'enrichir l'explication qu'on peut donner de faits mis en évidence par la quantification. Celle-ci ne permet pas toujours, en effet, de resituer les travailleurs ni dans leurs contextes de travail ni, plus largement, dans leurs conditions d'existence. Elle s'appuie aussi parfois sur des découpages préconstitués du travail et des travailleurs, plus ou moins juridiques, administratifs, savants. Dans la mesure où l'ethnographie s'attache à envisager le travail du point de vue de ses différents acteurs (gestionnaires, encadrement intermédiaire, travailleurs, etc.) en mettant au jour les catégories souvent multiples, contradictoires et instables au moyen desquelles ceux-ci perçoivent le travail et lui donnent sens, elle peut servir de remède contre l'ethnocentrisme savant ou administratif sous toutes ses formes (juridisme, économisme, légitimisme, intellectualisme, etc.). Elle peut en effet alimenter le travail critique d'explicitation des variables statistiques et de contrôle des interprétations que l'on peut en proposer (GoLLAC, 1997) qui est, comme l'ont rappelé Anne-Françoise et Serge, une caractéristique de la quantification du travail dans le système statistique public en France.

\section{Une fructueuse complémentarité des méthodes, ou la vertu des allers et retours entre les faits et leur interprétation}

Marie Cartier. La recherche sur la discrimination syndicale menée par Baptiste Giraud, Amaël MARChand et Étienne Penissat (2016, TE $\mathrm{n}^{\circ}$ 145) montre très bien cette possible complémentarité (ou mieux vaut-il dire « combinaison » pour ne pas colporter de fausses représentations d'une division a priori des objets et des échelles 
entre les méthodes ?), dont la principale condition est de respecter la logique de chaque méthode plutôt que de les mélanger (BEAUD, 1996b). En fait, il convient de ne pas confondre technique d'enquête et technique d'analyse et de respecter le type de raisonnement épistémologique associé à l'étude de cas ou à l'étude d'échantillon représentatif. Si l'on quantifie dans le cadre d'une étude de cas, alors on doit se cantonner à de la statistique descriptive sans verser dans la statistique inférentielle (GROs, 2017). Les traitements statistiques de l'enquête REPONSE mettent en évidence que c'est dans les grands établissements, ceux où les institutions représentatives du personnel sont les plus présentes et où les relations professionnelles sont de plus en plus encadrées juridiquement que paradoxalement le sentiment de discrimination ressenti par les syndicalistes est le plus fréquent. Comment l'expliquer ? Trois études de cas monographiques permettent tout d'abord de confirmer l'hypothèse selon laquelle la présence d'un contexte social plus souvent conflictuel dans les grands établissements y favorise non seulement l'existence d'un sentiment de discrimination syndicale mais surtout son expression. Les études de cas permettent aussi de comprendre que ce sentiment est pluridimensionnel et donc invitent à ne pas surinterpréter le fait statistique qui associe grands établissements et sentiment de discrimination syndicale. En l'absence de discrimination objective individuelle, le sentiment déclaré peut recouvrir une plainte plus collective face aux pratiques patronales, plus ou moins discrètes, d'entrave aux droits syndicaux. Il se nourrit par ailleurs de la comparaison et de la concurrence entre syndicats, ceux-ci faisant précisément l'objet d'une gestion plus visiblement différenciée de la part des directions dans les grands établissements. L'expression d'un sentiment de discrimination syndicale peut traduire enfin, autant qu'une situation objective de discrimination, une familiarisation militante au mode d'action juridique et à la catégorie de « discrimination », qui s'est précisément développée dans les grandes entreprises, notamment automobiles (HATZFELD, 2016, TE $\mathrm{n}^{\mathrm{o}}$ 145).

Anne-Françoise Molinié et Serge Volkoff. Nous avons aussi des réticences à user ici du terme de «complémentarité » entre les approches. Ce terme renvoie souvent à un rapport entre des approches de quantification, qui seraient à visée globale mais réductrice, et des approches qualitatives, qui saisiraient la complexité du réel dans des situations toujours singulières. Cette idée est parfois promue face aux inquiétudes ${ }^{2}$ que soulève « l'emprise du chiffre », avec la multiplication des champs qui font l'objet d'une mesure chiffrée. Cependant, raisonner en termes de complémentarité incite à conserver cette dichotomie entre qualitatif et quantitatif, avec un partage des rôles qui repose sur l'assignation à chacun de ces registres d'une échelle d'observation (individu vs collectif plus ou moins large) ou d'une temporalité (instantané $v s$ moyen ou long terme). Or

\footnotetext{
2. Inquiétudes ou réflexions dont témoigne la multiplication des numéros spéciaux ou dossiers de revues consacrés à ces thèmes depuis les années 2000. Par exemple : Genèses (2005), « Quantifier », $\mathrm{n}^{\circ} 58$; Revue française de socioéconomie (2010), «Les politiques de quantification », $\mathrm{n}^{\circ} 5 ;$ Terrains \& Travaux (2011), « Décrire et compter », $\mathrm{n}^{\circ} 19$; Sociologie et sociétés (2011), « La statistique en action» vol. 43, $\mathrm{n}^{\circ} 2$; Entreprises et histoire (2015), « La magie du chiffre », $\mathrm{n}^{\circ} 79$; Terrains \& Travaux (2016), « Mesurer pour prévenir ? », $\mathrm{n}^{\circ} 28$; Sociologie du travail (2016), « Le gouvernement par les indicateurs », vol. 58, $\mathrm{n}^{\circ} 4$; Revue française de socio-économie (2017), «Quantifier les qualités », nº 19.
} 
la «micro-statistique »- parfois sur quelques dizaines d'individus ou de situations de travail, ou sur des analyses de l'activité par séquences de quelques minutes, voire quelques secondes -s'est avérée précieuse, par exemple en ergonomie (PuEYo, 2000, TE $\mathrm{n}^{\circ} 84$ ). Symétriquement, des approches purement qualitatives ont fait la preuve de leur capacité à analyser, avec le recul nécessaire, les évolutions du travail dans l'ensemble d'un métier et sur plusieurs décennies - mais ce n'est pas à nous de développer cela ici.

La « complémentarité » est aussi souvent pensée en termes d'associations qui seraient nécessairement séquentielles. Les approches qualitatives (observations, entretiens) viendraient nourrir la préparation d'une enquête ou en éclairer les résultats ; ou encore, les données chiffrées fourniraient le cadrage pour des approches monographiques. Relèvent de cette démarche séquentielle les processus d'élaboration des enquêtes Conditions de travail, Sumer, etc., avec des comités scientifiques intégrant des chercheurs « de terrain », ou symétriquement les post-enquêtes lancées par des appels à projets autour de ces dispositifs statistiques. Ces combinaisons se sont avérées fructueuses, mais on peut aussi souligner l'intérêt d'articulations plus directes. C'est par exemple l'objectif de l'observatoire Evrest, que nous avons déjà mentionné. Celui-ci a été conçu d'emblée pour offrir « une infrastructure commune tout en ouvrant la possibilité d'usages diversifiés » (MOLINIÉ, LEROYER, 2011, § 35). Un dispositif de ce type permet de constituer des « objets-frontières », dotés d'une flexibilité interprétative précieuse aussi bien pour des chercheurs de diverses disciplines (BUCHMANN et al., 2018) que pour des médecins du travail dans leur pratique clinique.

Pour préciser notre propos, ajoutons que l'approche en termes de complémentarité n'a pas la même pertinence selon qu'on adopte une démarche de quantification à visée « explicative » ou « compréhensive » (Molinié, VolKoff, 2016). Dans le premier cas, il s'agit d'établir, de vérifier ou d'évaluer des liens de causalité entre, par exemple, telle caractéristique des conditions ou de l'organisation du travail et telles « conséquences » à plus ou moins long terme sur le résultat de l'activité de travail ou sur la santé ; dans cette perspective, il est logique de confier à l' analyse quantitative l'appréciation de l'ampleur de ces liens, et aux études qualitatives - de quelque discipline qu'elles relèvent - l'examen critique de leur caractère causal. Dans le second cas en revanche, l'objectif est d'embrasser l'ensemble des composantes de la situation de travail, en repérant leurs relations pour agir à bon escient ; cela suppose, selon nous, non d'attendre que la quantification et l'approche qualitative fournissent chacune, respectivement, ce qui « manquerait » à l'autre, mais de construire des articulations entre elles tout au long de la démarche de recherche ; cette deuxième perspective, dans laquelle se placent la plupart des travaux de notre réseau de recherche, a été particulièrement bien illustrée à notre avis par l'analyse d'une étude menée dans une fabrique de bottes par Nicole VÉzINA et Susan STOCK (2005). Si la dernière est épidémiologiste, la première est ergonome, mais son positionnement sur cette question des combinaisons d'approches nous semble faire largement écho à ce qu'explique Marie à propos des enquêtes ethnographiques. 


\section{Qu'avons-nous ainsi appris sur le travail depuis quarante ans?}

\section{Un travail qui expose toujours à des risques professionnels, s’intensifie et finit par user les corps}

Anne-Françoise Molinié et Serge Volkoff. Un premier résultat important des enquêtes statistiques portant sur le travail a été de montrer, non une évolution, mais une persistance : celle des expositions professionnelles pathogènes, au fil des décennies. Les efforts physiques importants, les environnements agressifs (le bruit, les toxiques, etc.), ou le travail de nuit par exemple, ne sont pas des contraintes ou nuisances en voie de disparition. De longue date, les enquêtes Conditions de travail ou Sumer ont remis en question l'idée selon laquelle les pénibilités physiques s'effaceraient au bénéfice d'un travail moins fatigant, moins pathogène et moins dangereux (HÉRAN-LEROY, SANDRET, 1996), et cela s'est confirmé au fil du temps (AlgaVA et al., 2014 ; BeQUE et al., 2017).

L'élargissement de l'empan temporel des interrogations à l'ensemble de la vie professionnelle (avec l'enquête Santé et itinéraire professionnel) a permis d'enrichir ce constat par l'inscription de ces contraintes dans les histoires professionnelles de différentes générations, montrant qu'elles ne reculent pas d'une génération à l'autre. Si les transformations structurelles de l'économie tendent plutôt à réduire le poids des catégories les plus exposées aux pénibilités physiques du travail, d'autres catégories de travailleurs (indépendants, professions intermédiaires et employés) déclarent de plus en plus souvent ces expositions (ROUXEL, VIRELY, 2012). Cette prise en compte du temps long a aussi permis de montrer que des expositions durables à ces contraintes physiques s'avèrent prédictives de fragilisations à long terme, tant dans l'emploi - avec des sorties précoces de l'emploi plus fréquentes avant l'âge de la retraite (WALTISPERGER, 2008) - qu'au regard de la santé - avec un surcroît de limitations fonctionnelles dans les activités de la vie quotidienne (COUTROT et al., 2010). En résumé, le travail continue à nuire à la santé.

Du côté des évolutions, un résultat majeur des enquêtes a certainement été la mise en évidence des formes et des effets de l'intensification du travail (GolLAC, 2005). Au-delà de l'idée répandue selon laquelle un travail plus intense se repérerait par une pression sur la production par unité de temps (l'« intensité-débit»), ces analyses ont attiré l'attention sur les formes nouvelles de l'intensification, avec la pénétration des logiques marchandes (réactivité par rapport au client, raccourcissement des délais, etc.) dans les univers industriels et, symétriquement, le développement de normes et de procédures de travail formalisées ainsi que d'autres formes de contraintes industrielles dans des univers jusque-là organisés sur le mode marchand, comme la grande distribution (GollaC, VolKOFF, 1996 ; AsKenazY et al., 2006). Ces types de contraintes existaient depuis longtemps dans le monde du travail, mais de façon en général disjointe, chacune d'elles prédominant dans un espace professionnel donné. 
Ce qui s'est révélé nouveau, c'est la progression de chacune de ces modalités et leur enchevêtrement, qui conduisent les travailleurs à s'ajuster à de multiples temporalités - temps du client, temps du produit, temps des collègues, etc. - qui ne recouvrent ni les mêmes exigences, ni les mêmes rythmes.

Ce cumul de contraintes industrielles, bureaucratiques et marchandes s'accompagne d'un accroissement significatif des pénibilités ressenties (postures, mouvements douloureux ou fatigants, port de charges lourdes, etc.), et du sentiment de n'avoir pas assez de temps pour faire correctement son travail. Mais ces derniers ne se traduisent pas systématiquement par une dégradation de la santé ni par un sentiment de « malheur au travail ». Au moins temporairement, une partie des salariés peut trouver dans l'intensification du travail une forme de stimulation et un investissement vécu comme positif (BAUDELOT, Gollac, 2003). L'intensification, qui peut aussi contribuer à infléchir et différencier les itinéraires professionnels (AMOSSÉ, GOLLAC, 2008 ; DE LARQUIER, REMILLON, 2008, $T E \mathrm{n}^{\circ}$ 113), participe de l'individuation de plus en plus forte des enjeux de santé selon les individus (VolKOFF, 2008). Parce qu'elles peuvent rendre compte à la fois des évolutions du travail et de la diversité des conditions de travail entre catégories de salariés, les approches quantitatives enrichissent la compréhension de cette diversité.

\section{Des transformations du travail et de l'emploi qui ont des résonances bien au-delà de l'univers professionnel}

Marie Cartier. Aux côtés de la statistique, l'ethnographie et les méthodes qualitatives ont également contribué à décrypter ce processus d'intensification du travail, en mettant notamment en évidence les formes renouvelées du travail subalterne, certes informatisé, fluidifié et au contact du marché, mais toujours aussi usant pour la santé, que ce soit aux caisses de la grande distribution (BERNARD, 2012) ou dans les entrepôts de la logistique (GABORIEAU, 2016). Mais l'ethnographie a aussi enrichi l'étude des transformations du travail lui-même. Dans son article fondateur paru dans Travail et Emploi et intitulé «L'ouvrière et le chef d'équipe ou comment parler du travail ? », M. PiALOuX (1995, $T E \mathrm{n}^{\circ}$ 62) réfutait ainsi l'idée partagée par nombre d'économistes, sociologues et politistes, selon laquelle les transformations du travail s'expliquaient principalement par l'organisation du travail et les politiques managériales conduites dans les entreprises : il affirmait qu' "il semble impossible de séparer les transformations technico-organisationnelles et ce qui relève des transformations (sociales, scolaires ou politiques...) qui se développent dans un autre "espace" que l'usine » (p. 39). Donnant une portée épistémologique au principe méthodologique consistant à suivre les travailleurs sur d'autres scènes que celles du travail, M. PIALOuX invitait à relier les transformations du travail aux aspirations et aux dispositions scolaires et sociales des travailleurs et à leur rapport au politique. Les transformations du travail ne s'imposent jamais d'elles-mêmes sans l'engagement de ceux qui les promeuvent ou les subissent : or comment rendre compte de ces engagements ? Dans Retour sur 
la condition ouvrière, S. BEAUd et M. PIALOUX (1999) ont mené une enquête ethnographique «longitudinale », pour emprunter un terme du vocabulaire statistique, c'est-à-dire une ethnographie qui permet les comparaisons dans le temps. Ce faisant, ils ont pu expliquer la déstructuration de la condition ouvrière à Peugeot-Sochaux par un faisceau de facteurs imbriqués : les nouvelles formes d'organisation du travail, mais aussi la différenciation du rapport au travail entre générations ouvrières inégalement scolarisées, placées dans des conditions d'emploi opposées (intérim/ contrat à durée indéterminée) et inégalement socialisées à la culture d'atelier. De nombreuses recherches à caractère ethnographique et historique ont ensuite retrouvé de tels processus dans d'autres contextes : dans l'industrie rurale (RENAHY, 2010) ou dans les services (y compris publics), par exemple dans la restauration rapide (PINTO et al., 2000, $T E \mathrm{n}^{\circ}$ 83). Ces travaux ont ainsi montré comment les nouvelles formes d'organisation de travail s'imposaient puis se pérennisaient par l'ajustement entre les nouvelles exigences productives (souvent désormais tout à la fois marchandes, bureaucratiques et industrielles, comme cela a été indiqué) et les propriétés sociales des travailleurs et, plus précisément, les dispositions liées à leurs trajectoires scolaires et professionnelles et à leurs âges.

De la même façon, l'ethnographie a contribué à mettre en question une approche uniquement juridique de la précarité en documentant la diversité de ses formes concrètes, qui tiennent parfois davantage au statut de l'entreprise qu'au contrat de travail (LinHART, MARUANI, 1982, TE $\mathrm{n}^{\circ} 11$ ). D'ailleurs, l'existence de salariés formellement stables mais confrontés sur la longue durée aux restructurations répétées de leur entreprise apparaît aussi habitée par l'incertitude (LoMBA, 2018). Et une même catégorie juridique d'emploi peut recouvrir des réalités très différentes selon le sexe des travailleurs, ou selon leur position dans le cycle de vie et leurs trajectoires professionnelles (KERGOAT, 1984, $T E \mathrm{n}^{\circ} 21$ ). L'intérim est une forme de précarité masculine qui fait l'objet d'usages sociaux différenciés : intérim d'insertion, de transition et de profession (FAURE-GUICHARD, 1999, TE $\mathrm{n}^{\circ}$ 78). En saisissant l'imbrication des transformations du travail et de l'emploi et des transformations sociales telles que l'allongement des scolarités et de la « jeunesse », ou la féminisation de la population active, l'ethnographie me semble avoir contribué à expliquer l'effritement de la société salariale de façon particulièrement réaliste, loin de l'intellectualisme d'explications se focalisant sur les idées et les actions politiques.

\section{L'observation des processus de socialisation professionnelle et du travail de service}

Marie Cartier. Contrairement aux États-Unis où la socialisation professionnelle a été étudiée dès les années 1950, cette dimension d'analyse est plus récente en France, qu'il s'agisse de la socialisation aux différents métiers ou aux différentes conditions d'emploi. Je pense que cette émergence est en partie liée à la diffusion de l'ethnographie, qui permet d'aborder finement les multiples contenus et mécanismes de socialisation professionnelle. Le numéro de Travail et Emploi sur la fabrication du 
soignant au travail dirigé en 2015 par Florent SCHEPENS et Emmanuelle ZoLESIO se fait bien l'écho de ce renouvellement théorique : la socialisation professionnelle peut s'étudier dans les lieux de formation, mais aussi dans les situations de travail ellesmêmes ; elle ne concerne pas moins les métiers du bas et du milieu de la hiérarchie de travail (aides-soignantes et infirmières) que ceux du haut (chirurgiens). Plusieurs thèses récentes consacrées au travail dans des petites entreprises illustrent aussi ce dynamisme de l'approche ethnographique de la socialisation professionnelle (DESPRAT, 2017 ; TRÉMEAU, 2017).

L'étude du travail dans les services ne se limite toutefois pas à la question de la socialisation, et c'est d'ailleurs un des apports de Travail et Emploi que d'avoir permis de comprendre d'autres enjeux, tels ceux spécifiquement posés par les transformations structurelles de l'économie. À la création de la revue, la sociologie du travail française était encore très centrée sur le monde ouvrier de la grande industrie alors même que la tertiarisation de l'emploi était en marche de longue date. Aux côtés des autres méthodes et disciplines, l'ethnographie du travail a depuis pleinement contribué à explorer le secteur des services. Elle en a mis au jour, contre l'insistance des rhétoriques professionnelles centrées sur «le relationnel », les dimensions manuelles et matérielles (ARBORIO 2001), y compris dans les métiers médicaux prestigieux (PENEFF, 1997) ou ceux dits de care (CARTIER, LECHIEN, 2012 ; AvrIL, 2014, 2018). Plusieurs recherches ethnographiques ont également établi que dans ce travail de service de plus en plus rationalisé, intense et individualisé, il n'en subsistait pas moins des formes d'appartenances collectives et de sociabilités au travail qui unissent et/ou qui divisent certes, mais qui toujours façonnent le rapport au travail, qui n'apparaît décidément jamais sous le regard de l'ethnographe comme strictement individuel (ALONZO, 1998, $T E \mathrm{n}^{\circ} 76$; CASTRA, 2004, TE $\mathrm{n}^{\circ} 97$; Avril, 2014). Et ce sont enfin les processus de recrutement des clients des services de santé (PenefF, 2000 ; Belorgey, 2013, TE $\mathrm{n}^{\mathrm{o}} 133$ ) ou d'accès à l'emploi (MARChAL, 1999, TE $\mathrm{n}^{\circ} 78$; Lima, Moulin, 2006, TE $\mathrm{n}^{\mathrm{o}} 105$ ) et l'influence des rapports de classe dans les relations de service (CARTIER, 2003 ; SerRe, 2012 ; MenouX, 2016) qui ont ainsi pu être étudiés.

\section{L'émergence des risques psychosociaux (RPS) : la statistique révélatrice des transformations du travail et du regard que la société porte sur lui}

Anne-Françoise Molinié et Serge Volkoff. Nous parlions plus haut des maux persistants du travail et de ses pénibilités physiques. Également emblématiques de la transformation du travail, et de façon en partie liée à la «tertiarisation » des champs de recherche que Marie vient de rappeler, les « facteurs psychosociaux de risques » se sont invités récemment dans les investigations statistiques en France, suite aux préconisations du rapport Nasse-Légeron ${ }^{3}$, dans un contexte de montée en puissance des débats sociaux et médiatiques sur les dégâts psychiques du travail. L'orientation des

3. Nasse P., Légeron P. (2008), Rapport sur la détermination, la mesure et le suivi des risques psychosociaux au travail, Paris, Ministère du Travail, des Relations sociales et de la Solidarité. 
approches quantitatives en ce domaine a été confiée à un Collège d'expertise, dont la qualité et l'originalité des travaux ${ }^{4}$ sont dues, comme le rappelle Michel GolLAC (2012, $T E \mathrm{n}^{0} 129$, p. 5), qui le présidait, « à la forte mobilisation du monde de la recherche pour répondre à ce qui lui apparaissait comme une demande sociale prioritaire », mais aussi à la disponibilité de l'appareil statistique qui avait en partie défriché ce domaine avant même que le débat social n'en amplifie l'intérêt. Il nous paraît important d'insister sur cet épisode dans l'évolution des approches quantitatives du travail en France, car il devrait marquer des orientations à venir, et il a soulevé des questions épistémologiques majeures.

Le Collège avait pour mission de préciser ce qui devait être mesuré et définir des critères de mesure à partir des connaissances théoriques et empiriques des membres, en examinant la littérature scientifique (nationale et internationale), les questionnaires existants, et par l'audition de personnalités scientifiques et d'acteurs sociaux. Cette réflexion partagée autour de la question de la quantification a été l'occasion de débats, le premier d'entre eux concernant la signification à donner à ce terme de « risques psychosociaux » pour que l'on puisse mesurer ces risques en s'appuyant sur des connaissances scientifiques. Comme le rappellent Marceline BodiER et Loup WOLFF (2018, p. 31), « il n'y avait pas, en l'état actuel de l'art, de consensus sur la façon de concevoir et de traiter les risques psychosociaux au travail », et « encore moins une théorie universelle et unifiée au sujet de ces risques ». Les choix effectués ont de fait conduit à plusieurs déplacements. Le premier a consisté à s'écarter de deux approches fortement présentes dans les représentations de ces RPS : d'une part, une tendance à individualiser les problèmes - renvoyant à une supposée fragilité des personnes impliquées et à des modes de traitement par l'accompagnement et le soutien psychologique ; d'autre part, une grille de lecture amenant à considérer les RPS comme une « exposition », dont on pourrait mesurer le degré, comme on le fait pour un niveau de bruit ou un toxique, et qui apparaîtrait, au moins en partie, comme inévitable. Le Collège a proposé à l'inverse une acception large des RPS, comme « risques pour la santé mentale, physique et sociale, engendrés par les conditions d'emploi et les facteurs organisationnels et relationnels susceptibles d'interagir avec le fonctionnement mental ». Il a considéré que l'accent devait être mis sur les facteurs de RPS, qui relèvent principalement de l'organisation du travail et de l'entreprise, mais aussi de caractéristiques intrinsèques des métiers et de certains aspects des parcours professionnels - facteurs vis-à-vis desquels il y avait des possibilités d'agir. Le Collège a également choisi de se centrer sur des « facteurs » dont les effets sur la santé étaient bien établis et de ne pas limiter a priori le nombre d'indicateurs à retenir (contrairement à la préconisation du rapport Nasse-Légeron d'aller vers « un » indicateur de RPS).

Nous ne développerons pas ici les choix qui ont été faits (BODIER, WOLFF, 2018) de retenir une quarantaine d'indicateurs, dont certains étaient déjà disponibles dans les enquêtes existantes, et qui ont été regroupés en six grandes dimensions : l'intensité

4. Repris ensuite dans un numéro spécial de TRAVAIL ET EMPLOI (2012, n $\left.{ }^{\circ} 129\right)$ et dans un ouvrage récent (BoDIER, WOLFF, 2018). 
du travail et le temps de travail ; les exigences émotionnelles ; le manque d'autonomie et de marges de manœuvre ; les rapports sociaux au travail ; les conflits de valeur ; l'insécurité de la situation de travail. Si nous avons insisté sur l'originalité de cette démarche, c'est qu'elle nous semble illustrer le rôle de « langage-carrefour » que peut jouer la statistique, selon l'acception proposée par Alain DESROSIÈRES (1993, p. 355) : «Une ressource argumentative et décisionnelle souple, susceptible d'usages variés, dans des constructions foncièrement différentes les unes des autres »-constructions aussi différentes, selon nous, que peuvent l'être les situations de travail. Et comme l'indique Yves CLOT (2010, p. 127), psychologue du travail et théoricien de la clinique de l'activité, dont l'appréciation des travaux du Collège, et des analyses chiffrées en général, est nuancée : «j'ai pu mesurer à quel point la mise en débat de données chiffrées dans un collectif professionnel peut devenir une source de controverse et de pensée ; mais dans ce cas le chiffre est pris comme objet d'une analyse en vue d'un diagnostic qu'il ne contient pas en puissance ».

\section{L'ampleur et les formes de la «division sexuelle du travail »}

Marie Cartier. Je voudrais mentionner un dernier apport, non pas de l'ethnographie seule, mais de la combinaison entre ethnographie et statistiques. Il se situe du côté de l'étude de la « division sexuelle du travail » (KERGOAT, 2005), c'est-à-dire des formes, variables mais permanentes dans l'histoire, de séparation et de hiérarchisation du travail attribué à chaque sexe. C'est devenu un objet d'étude privilégié des nouvelles générations de sociologues du travail. La revue Travail et Emploi y a contribué dès le tout début des années 1980, non seulement par des analyses statistiques des enquêtes Conditions de travail mais aussi par des enquêtes plus qualitatives. Par exemple, un article publié en 1983 interrogeait les spécificités du rapport au travail des femmes ouvrières et employées au moyen d'entretiens biographiques approfondis, parfois croisés au sein d'une même famille. Il montrait que ces femmes, tout en étant très attachées à leur emploi, avaient besoin de l'appui domestique de leur propre mère pour se maintenir sur le marché du travail et qu'elles tendaient à adapter leur activité professionnelle non seulement aux contraintes objectives de leur travail domestique mais aussi aux représentations qu'elles s'en faisaient (DAUNE-RICHARD, 1983, TE $\mathrm{n}^{\circ} 17$ ). Autre exemple, une enquête qui a montré que l'usure prématurée de ces ouvrières et employées - que les politiques de prévention peinent à saisir - apparaissait davantage liée à la pénibilité vécue au travail et hors travail qu'à l'exposition à des nuisances (ThÉBAud-MonY, 1992, TE $\mathrm{n}^{\circ}$ 54).

Pour ce qui est de la ségrégation sexuée des emplois et des conditions de travail - mise en évidence dès les années 1970 dans le monde ouvrier -, l'approche statistique a bien évidemment été fondamentale. Mais là encore, si l'on reprend les premiers travaux, on peut voir que la combinaison entre statistiques et approches plus qualitatives et contextualisées a permis d'enrichir l'explication de la façon dont la ségrégation se construit non seulement par la socialisation différentielle de sexe mais aussi par la force 
des stéréotypes de genre chez les employeurs et les gestionnaires du travail (KERGOAT, [1978] 2012 ; Maruani, NiCOLE-DranCourT, 1989 ; GollaC, VolKOFF, 2002). L'emploi ne fait pas que refléter les inégalités entre les sexes mais participe à leur reproduction : l'ethnographie a contribué à éclairer le « comment» de ce processus. En contexte de mixité, ce sont souvent les carrières et les tâches qui, derrière un même intitulé de poste, se révèlent sexuellement différenciées - une différence qui n'est pas forcément d'avance rendue visible par les découpages officiels et que l'ethnographie a pu aider à repérer. Les hommes qui exercent des activités pensées comme « féminines » bénéficient d'avantages en termes de liberté de mouvement mais aussi de sociabilité, de sexualité et de promotion (Louey, SCHÜTZ, 2014, TE n ${ }^{\circ} 140$ ), la situation des femmes dans des métiers d'hommes étant bien loin d'être symétrique. Un numéro récent de Travail et Emploi a en ce sens mis en chantier l'étude des conséquences du renouveau du travail indépendant sur la division sexuée du travail (ABDELNOUR et al., 2017, TE $\mathrm{n}^{\circ}$ 150).

\section{Quel programme pour les années à venir ?}

\section{Poursuivre l'analyse de thématiques « classiques » : le genre, la santé et les conditions de travail}

Marie Cartier. Je me contenterai d'esquisser quelques suggestions personnelles à partir des apports dégagés : il ne s'agit pas de dresser un programme de recherche ancré dans un bilan exhaustif des résultats de la sociologie et de l'ethnographie du travail mais juste d'essayer de cerner des questions actuelles appelant tout particulièrement une approche ethnographique. L'étude de la différenciation sexuée des pratiques de travail mérite d'être poursuivie. La recherche d'Anne PAILLET et Delphine SERRE (2014) sur les juges des enfants propose des pistes originales, qui invitent à se garder de tout différentialisme a priori. L'observation du travail sur les enfants en audience fait en effet apparaître des magistrates froides et distantes et des magistrats maternant les enfants. Ce n'est pas que le genre ne joue pas, mais plutôt qu'il n'agit ni de façon isolée, ni directement et uniformément : l'effet du genre sur les pratiques de travail peut être freiné ou redoublé par les trajectoires scolaires et professionnelles et médié par le rapport aux normes éducatives psychologisées ou aux stéréotypes de genre. Quels types de contextes professionnels et de trajectoires favorisent le cumul des modes d'action du genre et fabriquent des pratiques de travail sexuées ? Lesquels, au contraire, favorisent des pratiques de travail mixtes? L'ethnographie, dans la mesure où elle permet de tenir compte des façons de travailler, des contextes professionnels et des multiples dimensions des trajectoires, ainsi que des médiations concrètes par lesquelles agissent les facteurs de différenciation des pratiques, apparaît particulièrement adaptée pour travailler de telles questions.

J'évoquerai aussi le domaine de recherche dynamique que constitue la santé au travail. Christelle Avril et Pascal Marichalar (2016), dans le cadre d'un dossier 
récent de Travail et Emploi $\left(\mathrm{n}^{\circ} 147\right)$ consacré aux multiples voies par lesquelles risques et pénibilités professionnelles pénètrent la sphère familiale, proposent de réinvestir la perspective féministe des années 1970 consistant à articuler sphère du travail et sphère de la famille pour renouveler l'étude de la santé au travail des hommes et des femmes. En quoi le travail domestique et ses pénibilités sont-ils affectés par les conditions du travail rémunéré ? Dans quelle mesure la reconnaissance des atteintes professionnelles à la santé des hommes dépend-elle du fait qu'ils ont une conjointe et de la trajectoire sociale et professionnelle de cette dernière ? Et ne doit-on pas se donner les moyens de repérer aussi les mobilisations des hommes pour faire reconnaître les atteintes professionnelles à la santé de leurs conjointes et explorer leurs ressorts ? L'ethnographie pouvant être définie par son ambition à circuler entre les scènes sociales, elle me semble constituer une méthode particulièrement adaptée pour explorer de telles pistes.

Anne-Françoise Molinié et Serge Volkoff. Comme l'indique Marie Cartier, et de façon cohérente avec nos propos précédents, nous tenons à souligner que les approches quantitatives ne désignent pas des pistes de recherche qu'elles seraient à même d'explorer seules, sans que se déploient des analyses qualitatives par ailleurs. Cela étant, une première mission qu'on peut confier à « la quantification », c'est d'inviter à ne pas délaisser les hypothèses ou les pistes de recherche anciennes, celles qui concernent des aspects du travail existant certes de longue date, mais dont les statistiques ne cessent de confirmer l'actualité. On l'a dit à propos des contraintes et nuisances du travail manuel, mais il en va de même s'agissant des horaires décalés, des tâches répétitives, des ambiances physiques agressives ou des risques d'accidents : il y a toujours lieu de mieux comprendre pourquoi le progrès technique, ou la disparition d'emplois parmi les plus contraignants, ne s'accompagne pas - selon ce qu'en disent les tendances indiquées par ces enquêtes (MAUROuX, 2016) - d'une amélioration d'ensemble dans ces domaines. Il y a toujours lieu aussi d'apprécier comment ces aspects contribuent à des mécanismes d'usure professionnelle et d'exclusion précoce de l'emploi et, ce faisant, aux inégalités sociales de santé. Le caractère «pesant » des démarches statistiques a ici un mérite : en posant de façon récurrente au fil du temps des questions immuables, et en les posant à tous, elles font preuve d'une inertie bienfaisante et contrarient utilement les effets de mode. On rejoint ici les propos de Michel GollaC (1998), qui considère que la routine est aussi essentielle au statisticien qu'à un investigateur policier. Les facteurs psychosociaux de risques, on l'a dit, se sont invités plus récemment dans les investigations statistiques en France, et là aussi, la poursuite des recherches engagées constitue par elle-même un objectif légitime. Les travaux du Collège d'expertise ont permis d'en préciser et articuler les composantes. Cela favorise des approches quantitatives qui ne soient pas trop réductrices, qui prêtent attention à la diversité des aspects du travail (l'intensité, les exigences émotionnelles, l'autonomie, etc.), comme à celle des sensations ou des troubles de santé éprouvés (la fatigue, les craintes, les tensions, etc.). La série des enquêtes nationales sur les conditions de travail a commencé à en tirer parti (Coutrot, 2018), de même que certains dispositifs en entreprises. 


\section{Explorer des questions « nouvelles » croisant travail et emploi}

Anne-Françoise Molinié et Serge Volkoff. Nous sommes tentés de retenir deux questions témoignant d'un renouvellement des objets de recherche, et de les relier : d'une part, le développement d'analyses à caractère diachronique, attentives aux dimensions de moyen et long termes dans les trajectoires individuelles et aux dynamiques qui s'y révèlent ; d'autre part, le décloisonnement des questions d'emploi et de travail. En suggérant cette double orientation, nous faisons écho aux résultats, y compris quantitatifs, de recherches dans plusieurs disciplines des sciences sociales. Ceux-ci suggèrent que les itinéraires professionnels sont appelés à être de moins en moins continus et stables (AMOSSÉ, GollaC, 2008, TE n ${ }^{\circ} 113$; RouXel, VireLY, 2012). Ils soulignent aussi que l'intensification produit - et se trouve elle-même accrue par - un régime de changements au rythme soutenu (WolfF et al., 2015 ; GreENAN, MAIRESSE, 2006).

De cette évolution peut résulter une forme de « soustraction» (GAUDART, 2015) du passé et du futur de celles et ceux qui la subissent. Les possibilités de convoquer son expérience s'en trouvent restreintes, ce qui limite les possibilités d'échafauder un avenir. Ce «présentisme », cet enfermement dans l'instant, constitue un facteur de vulnérabilité dont les effets varient selon les populations concernées. Les travailleurs âgés peuvent perdre pied parce que ce contexte rend inaccessibles les ressources de leur expérience. Les jeunes voient s'amoindrir leurs possibilités d'apprentissage. Les travailleurs qui souffrent de problèmes de santé se trouvent marginalisés quand font défaut les possibilités de développer des stratégies individuelles et collectives qui préserveraient leur bien-être et leur efficience. Cette tendance n'est ni systématique ni inéluctable. Elle devrait cependant constituer, à notre avis, une thématique majeure pour des recherches à venir, que les enquêtes statistiques, interprofessionnelles ou en entreprises, pourraient alimenter utilement. Des dispositifs existent pour cela, comme le montre l'introduction de questions sur les parcours dans les enquêtes nationales sur les conditions de travail (METTE, 2018), qui, en outre, comportent à présent un volet organisé en panel. L'observatoire Evrest, que nous avons mentionné à plusieurs reprises ici, ouvre aussi des possibilités d'analyses longitudinales, même s'il s'agit là d'un nombre plus limité de questions. La réédition de l'enquête SIP, réalisée en 2006 et 2010, n'est pas envisagée à notre connaissance ; cela pourrait justifier de déployer de nouveaux outils, ou d'aménager des outils existants, afin de progresser dans l'analyse des différentes formes de mobilité, internes ou externes au marché du travail, au moment où elles surviennent, plutôt que de manière rétrospective (HÉLARDOT, 2006).

Il est tentant d'élargir cette orientation à l'articulation entre le parcours professionnel et d'autres sphères de vie, objet d'investigation usuel de l'ethnographie comme l'a développé Marie Cartier. Les enjeux personnels et sociaux des mutations qu'on vient d'évoquer dépendent en effet de la place que les individus assignent à leur vie de travail, aux côtés de leurs autres activités, leurs autres contraintes ou investissements. Des recherches en sociologie ont attiré l'attention sur l'idée que les femmes et les hommes adopteraient une conception de plus en plus « polycentrique » de l'existence 
(MÉDA, VENDRAmin, 2013). Non qu'il s'agisse de prendre ses distances à l'égard du travail, mais plutôt de rendre possible un engagement dans plusieurs domaines sans sacrifier aucun d'eux. Se manifeste alors un souci de cohérence entre les différentes sphères de la vie, cohérence organisationnelle mais aussi cohérence en termes de valeurs : en particulier, la menace du chômage apparaîtrait de moins en moins comme un motif suffisant pour accepter un travail qui irait à l'encontre des valeurs personnelles. Cette perspective, brossée ici à grands traits, se prête évidemment à des investigations - entre autres - quantitatives. Elles ont débuté et ont d'ailleurs contribué à l'accréditer. Cependant, le champ des analyses possibles reste vaste. Celles-ci pourraient faire écho, à vingt ans de distance, aux questions que soulevaient Luc BOLTANSKI et Ève CHIAPELLO (1999) à propos des mutations dans les principes de management, et de leur congruence plus ou moins avérée avec une aspiration accrue à l'autonomie chez les individus. On peut aujourd'hui essayer d'apprécier les convergences ou les discordances entre des modèles de parcours plus hachés et moins prévisibles et des modèles de vie orientés vers de nouveaux équilibres. Les statistiques nationales ou européennes comme les enquêtes en entreprises pourraient contribuer à instruire cette question.

Marie Cartier. Les recompositions du capitalisme, de l'industrie à la finance, tournées vers l'enrichissement (BOLTANSKI, ESQUERRE, 2017) ont favorisé l'essor de nouvelles activités entre mondes professionnel et domestique et la question des frontières du travail connaît une nouvelle actualité. Dans la période historique que nous vivons, il convient d'étudier le travail sous toutes ses formes (indépendant/salarié, rémunéré/gratuit, producteur de valeur/moralisateur, familial/marchand, légal/illégal, etc.) sachant que celles-ci se combinent souvent aujourd'hui d'une façon inattendue et complexe (SimONET, 2018) et défient ainsi toutes les oppositions binaires telles celle entre amateur et professionnel (FLEURIEL, SCHOTTÉ, 2016) ou celle entre famille et marché, «mondes (supposés) hostiles » (ZeLIZER, 2008, p. 18). Or, comme je l'ai déjà souligné, l'ethnographie est particulièrement appropriée pour saisir les activités économiques indécises, non encore classées et juridicisées, qui, par exemple, relèvent, selon le point de vue considéré, du travail ou du loisir. Le «travail à-côté » dont Florence WEBER ([1989] 2009) a exploré la face ouvrière et masculine connaît ainsi d'intéressantes métamorphoses à la fois dans ses formes et significations concrètes et dans le profil social de ses pratiquants. Il serait aujourd'hui moins séparé de l'emploi principal qu' autrefois, en lien avec la diffusion des outils numériques d'autoproduction et d'intermédiation (Flichy, 2017). Derrière l'essor des vide-greniers et du Bon Coin ne se développe-t-il pas dans nombre de ménages de divers groupes sociaux une activité de ventes et d'achats intéressante à étudier ? À partir du cas du travail qui se déploie sur les plateformes collaboratives (BEAUVISAGE et al., 2018) ou sur les réseaux sociaux ${ }^{5}$, c'est la question des formes et ressorts sociaux de la mise au travail des internautes par les entreprises qui est désormais posée.

5. Joseph Godefroy mène dans le cadre d'une thèse de sociologie une recherche sur les usages économiques des réseaux sociaux à partir d'une enquête sur les «influenceurs » dans le secteur du fitness et de la cuisine (Cens, université de Nantes). 


\section{BibLIOGRAPHIE}

Abdelnour S., Bernard S., Gros J. (2017), « Genre et travail indépendant. Divisions sexuées et places des femmes dans le non-salariat. Introduction », Travail et Emploi, ${ }^{\circ}{ }^{150, p p . ~ 5-~} 23$. Algava É., Davie E., Loquet J., Vinck L. (2014), « Conditions de travail. Reprise de l'intensification du travail chez les salariés », Dares analyses, $n^{\circ} 049$.

Alonzo P. (1998), « Les rapports au travail et à l'emploi des caissières de la grande distribution. Des petites stratégies pour une grande vertu », Travail et Emploi, n ${ }^{\circ}$ 76, pp. 37-51.

Amossé T., Bloch-London C., WolfF L. (dir.) (2008), Les Relations sociales en entreprise. Un portrait à partir des enquêtes Relations professionnelles et négociations d'entreprise, REPONSE 1992-1993, 1998-1999, 2004-2005, Paris, La Découverte.

Amossé T., Gollac M. (2008), «Intensité du travail et mobilité professionnelle », Travail et Emploi, $\mathrm{n}^{\mathrm{o}} 113$, pp. 59-73.

Arborio A.-M. (2001), Un personnel invisible : les aides-soignantes à l'hôpital, Paris, Anthropos.

Askenazy P., Cartron D., De Coninck F., Gollac M. (coords.) (2006), Organisation et intensité du travail, Toulouse, Octarès.

Avril C. (2014), Les Aides à domicile. Un autre monde populaire, Paris, La Dispute.

Avril C. (2018), « Sous le care, le travail des femmes de milieux populaires. Pour une critique empirique d'une notion à succès », in Maruani M. (dir.), Je travaille, donc je suis. Perspectives féministes, Paris, La Découverte, pp. 205-216.

Avril C., Cartier M., Serre D. (2010), Enquêter sur le travail. Concepts, méthodes, récits, Paris, La Découverte.

Avril C., Marichalar P. (2016), «Quand la pénibilité du travail s'invite à la maison. Perspectives féministes en santé au travail. Introduction », Travail et Emploi, n ${ }^{\circ}$ 147, pp. 5-26.

Baudelot C., Gollac M. (2003), Travailler pour être heureux? Le bonheur et le travail en France, Paris, Fayard.

BEAud S. (1996a), " "Stage” ou formation ? Les enjeux d'un malentendu. Notes ethnographiques sur une mission locale de l'emploi », Travail et Emploi, n ${ }^{\circ}$ 67, pp. 67-89.

BEAUd S. (1996b), « L'usage de l'entretien en sciences sociales. Plaidoyer pour l'“entretien ethnographique" », Politix, n ${ }^{\circ} 35$, pp. 226-257.

BEAUD S. (1999), «Un cas de sauvetage social : histoire d'une “jeune précaire” racontée par un conseiller de mission locale », Travail et Emploi, n ${ }^{\circ}$ 80, pp. 77-89.

Beaud S., Pialoux M. (1999), Retour sur la condition ouvrière. Enquête aux usines Peugeot de Sochaux-Montbéliard, Paris, Fayard.

BeAud S., Weber F. (2012), « Le raisonnement ethnographique », in Paugam S. (dir.), L'Enquête sociologique, $2^{\mathrm{e}}$ éd., Paris, Presses universitaires de France, pp. 223-246. 
Beauvisage T., Beuscart J.-S., Mellet K. (2018), « Numérique et travail à-côté. Enquête exploratoire sur les travailleurs de l'économie collaborative », Sociologie du travail [en ligne], vol. 60, n 2. https://doi.org/10.4000/sdt.1984.

Belorgey N. (2013), « Pourquoi attend-on aux urgences? Un indicateur du New Public Management aux prises avec la réalité hospitalière », Travail et Emploi, $\mathrm{n}^{\circ} 133$, pp. 25-38.

Beque M., Mauroux A., Baradji E., Dennevault C. (2017), « Quelles sont les évolutions récentes des conditions de travail et des risques psychosociaux?», Dares analyses, $\mathrm{n}^{\circ} 082$.

Bernard L. (2017), La Précarité en col blanc. Une enquête sur les agents immobiliers, Paris, Presses universitaires de France.

BERnARD S. (2012), Travail et automatisation des services. La fin des caissières ?, Toulouse, Octarès.

Béroud S., Denis J.-M., Giraud B., Desage G., Carlier A., Pélisse J. (2008), « Une nouvelle donne ? Regain et transformation des conflits au travail », in Amossé T., Bloch-London C., Wolff L. (dir.), Les Relations sociales en entreprise. Un portrait à partir des enquêtes Relations professionnelles et négociations d'entreprise, REPONSE 1992-1993, 1998-1999, 2004-2005, Paris, La Découverte, pp. 223-255.

Bevort A., Jobert A., Lallement M., Mias A. (dir.) (2011), Dictionnaire du travail, Paris, Presses universitaires de France.

Bodier M., WolfF L. (2018), Les Facteurs psychosociaux de risque au travail : d'après le rapport d'expertise sur le suivi statistique des risques psychosociaux au travail présidé par Michel Gollac, Toulouse, Octarès.

Boltanski L., Chiapello È. (1999), Le Nouvel Esprit du capitalisme, Paris, Gallimard.

BOLTANSKi L., ESQUERRE A. (2017), Enrichissement : une critique de la marchandise, Paris, Gallimard.

Bourgois P. (2013), En quête de respect. Le crack à New York, éd. revue et augmentée, Paris, Seuil.

Buchmann W., Mardon C., Volkoff S., Archambault C. (2018), « Peut-on élaborer une approche ergonomique du "temps long" ? Une étude des douleurs articulaires liées au travail, dans une grande entreprise », Perspectives interdisciplinaires sur le travail et la santé [en ligne], $\mathrm{n}^{\mathrm{o}} 20-1$. https://doi.org/10.4000/pistes.5565.

CARTIER M. (2001), « Nouvelles exigences dans les emplois d'exécution des Services publics », Genèses, n ${ }^{\circ}$ 42, pp. 72-91.

CARTIER M. (2003), Les Facteurs et leurs tournées. Un service public au quotidien, Paris, La Découverte.

CARTIER M., LeChiEn M.-H. (2012), « Vous avez dit "relationnel” ? Comparer des métiers de service peu qualifiés féminins et masculins », Nouvelles Questions féministes, vol. 31, $\mathrm{n}^{\circ} 2$, pp. 32-48.

CASTRA M. (2004), « Faire face à la mort : réguler la "bonne distance" soignants-malades en unité de soins palliatifs », Travail et Emploi, n ${ }^{\circ}$ 97, pp. 53-64. 
CÉZArd M., Dussert F., Gollac M. (1992), « Taylor va au marché. Organisation du travail et informatique », Travail et Emploi, $\mathrm{n}^{\circ}$ 54, pp. 4-19.

Chapoulie J.-M. (2000), « Le travail de terrain, l'observation des actions et des interactions, et la sociologie », Sociétés contemporaines, ${ }^{\circ}$ 40, pp. 5-27.

Clot Y. (2010), Le Travail à cæur : pour en finir avec les risques psychosociaux, Paris, La Découverte.

Collectif Rosa Bonheur (2017), « Des “inactives” très productives. Le travail de subsistance des femmes de classes populaires ». Tracés. Revue de sciences humaines [en ligne], $\mathrm{n}^{\circ} 32$. https://doi.org/10.4000/traces.6862.

Coutrot T. (2018), « Travail et bien-être psychologique. L'apport de l'enquête CT-RPS 2016 », Document d'études, $\mathrm{n}^{\circ} 217$, Dares.

Coutrot T., Rouxel C., Bahu M., Herbet J.-B., Mermilliod C. (2010), « Parcours professionnels et état de santé » Premières Synthèses, nº01, Dares, Drees.

CURIE J. (1995), « Qu'est-ce qu'un bon chiffre ? », in Dares, L'Usage des méthodes statistiques dans l'étude du travail. Journée-débat du 19 janvier 1994, Paris, La Documentation française, pp. 25-29.

DAune-Richard A.-M. (1983), « Travail professionnel et travail domestique : le travail et ses représentations au sein de lignées féminines », Travail et Emploi, $\mathrm{n}^{\circ}$ 17, pp. 49-55.

DEFFONTAINeS P. (2018), «Survivre » 25 ans après la chute de l'URSS : classes populaires et marchés dans les espaces ruraux ukrainiens, thèse de doctorat en sociologie, démographie université de Bourgogne Franche-Comté.

DESPRAT D. (2017), "Qu'est-ce qu'on vous fait aujourd'hui ? » Un éthos professionnel des coiffeurs. Entre travail émotionnel, relation de service et dispositions genrées et de classe. Le cas des coiffeurs, thèse de doctorat en sociologie, université Paris Nanterre.

Desrosières A. (1993), La Politique des grands nombres. Histoire de la raison statistique, Paris, La Découverte.

Dodier N. (1990), Jugements médicaux, entreprises et protocoles de codage. La contribution des médecins du travail à l'enquête SUMER sur les risques professionnels, Paris, La Documentation française.

Dodier N. (1993), L'Expertise médicale. Essai de sociologie sur l'exercice du jugement, Paris, Métailié.

Dodier N., BASZANGer I. (1997), « Totalisation et altérité dans l'enquête ethnographique », Revue française de sociologie, vol. 38, $\mathrm{n}^{\circ}$ 1, pp. 37-66.

Dufy C., Weber F. (2007), L'Ethnographie économique, Paris, La Découverte.

FAURE-Guichard C. (1999), «Les salariés intérimaires, trajectoires et identités », Travail et Emploi, $\mathrm{n}^{\mathrm{0}} 78$, pp. 1-20.

FLeuriel S., SCHOTTÉ M. (2016), « Dépasser l'alternative amateurs/professionnels. Programme pour une histoire sociale des sportifs au travail. Éditorial », Le Mouvement social, $\mathrm{n}^{\mathrm{0}} 254$, pp. 3- 12. 
Flichy P. (2017), Les Nouvelles Frontières du travail à l'ère numérique, Paris, Seuil.

Frigul N., Bretin H., Metenier I., Aussel L., Thébaud-Mony A. (1993), « Atteintes à la santé et exclusion professionnelle. Une enquête auprès de 86 femmes en chômage de longue durée », Travail et Emploi, n ${ }^{\circ} 56$, pp. 34-44.

Gaborieau D. (2016), Des usines à colis. Trajectoire ouvrière des entrepôts de la grande distribution, thèse de doctorat en sociologie, université Paris-I Panthéon-Sorbonne.

GAudART C. (2015), « Le temps soustrait », in Thébaud-Mony A., Davezies P., Vogel L, Volkoff S. (dir.), Les Risques du travail Pour ne pas perdre sa vie à la gagner, Paris, La Découverte, pp. 196-206.

Giraud B., Marchand A., Penissat É. (2016), « Le sentiment de discrimination des représentants du personnel. Une étude à partir des données statistiques et monographiques liées à l'enquête REPONSE », Travail et Emploi, n 145, pp. 87-119.

Godechot O. (2005), Les Traders. Essai de sociologie des marchés financiers, nouv. éd., Paris, La Découverte.

Gollac M. (1997), « Des chiffres insensés ? Pourquoi et comment on donne un sens aux données statistiques », Revue française de sociologie, vol. 38, n 1, pp. 5-36.

Gollac M. (1998), À marches forcées? Contribution à l'étude des changements du travail, mémoire pour l'habilitation à diriger des recherches, université Paris-VIII.

Gollac M. (2005), «L'intensité du travail. Formes et effets », Revue économique, vol. 56, $\mathrm{n}^{\mathrm{o}} 2$, pp. 195-216.

GollaC M. (2012), « Les risques psychosociaux au travail : d'une "question de société" à des questions scientifiques. Introduction », Travail et Emploi, $\mathrm{n}^{\circ} 129$, pp. 5-10.

Gollac M., Volkoff S. (1996), «Citius, altius, fortius. L'intensification du travail », Actes de la recherche en sciences sociales, $\mathrm{n}^{\circ} 114$, pp. 54-67.

Gollac M., Volkoff S. (2002), « La mise au travail des stéréotypes de genre. Les conditions de travail des ouvrières », Travail, genre et sociétés, $n^{\circ} 8$, pp. 25-53.

Gollac M., Volkoff S. (2006), « La santé au travail et ses masques », Actes de la recherche en sciences sociales, $\mathrm{n}^{\circ} 163$, pp. 5-17.

Gollac M., VOLKOFF S. (2010), « Mesurer le travail. Une contribution à l'histoire des enquêtes françaises dans ce domaine », Document de travail, n 127, Centre d'études de l'emploi.

GreEnAN N., MAIRESSE J. (2006), «Les changements organisationnels, l'informatisation des entreprises et le travail des salariés. Un exercice de mesure à partir de données couplées entreprises/salariés », Revue économique, vol. 57, nº 6, pp. 1137-1175.

Gros J. (2017), «Quantifier en ethnographe. Sur les enjeux d'une émancipation de la représentativité statistique », Genèses, n 108, pp. 129-147.

Guiho-Bailly M.-P., Bertin C., Dubré J.-Y., Lancien N., Machefer J., Parent D. (2009), « Rapport subjectif au travail : sens des trajets professionnels et construction de la santé. Tomes 1 et 2 », Document de travail, $\mathrm{n}^{\circ}$ 95, série «Études et recherches », Drees. 
Guiho-Bailly M.-P., RoQuelaure Y. (2013), « Une post-enquête qualitative dans la validation technique de l'enquête statistique "Santé, itinéraire professionnel" : principes et méthode ", Santé publique, vol. 25, n 3, pp. 271-279.

HATZFELD N. (2016), « Une lutte syndicale exemplaire chez Peugeot-Sochaux (1995-2000). La remise en cause d'un système discriminatoire », Travail et Emploi, n 145, pp. 173-196.

HÉLARDOT V. (2006), « Parcours professionnels et histoires de santé : une analyse sous l'angle des bifurcations », Cahiers internationaux de sociologie, $\mathrm{n}^{\circ}$ 120, pp. 59-83.

Héran-Leroy O., SANDret N. (1996), « Expositions aux contraintes et nuisances dans le travail », Premières Synthèses, Dares, n 42-1.

HERLIN-GIRET C. (2019), Rester riche. Enquête sur les gestionnaires de fortune et leurs clients, Lormont, Le Bord de l'eau.

Jounin N. (2009), Chantier interdit au public. Enquête parmi les travailleurs du bâtiment, Paris, La Découverte.

KERGOAT D. (1984), «Les femmes et le travail à temps partiel : une relation multiforme et complexe au temps travaillé », Travail et Emploi, $\mathrm{n}^{\circ} 21$, pp. 7-21.

KERGOAT D. (2005), « Rapports sociaux et division du travail entre les sexes », in Maruani M. (dir.), Femmes, genre et sociétés. L'état des savoirs, Paris, La Découverte, pp. 94-101.

KergOAT D., ([1978] 2012), «"Ouvriers = ouvrières" ? Propositions pour une articulation théorique de deux variables : sexe et classe sociale », in Kergoat D., Se battre, disent-elles, Paris, La Dispute, pp. 33-62.

LARQUIER G. (DE), REMILLON D. (2008), «Assiste-t-on à une transformation uniforme des carrières professionnelles vers plus de mobilité ? Une exploitation de l'enquête Histoire de vie », Travail et Emploi, ${ }^{\circ} 113$, pp. 13-30.

Linhart D., Maruani M. (1982), «Précarisation et déstabilisation des emplois ouvriers. Quelques hypothèses », Travail et Emploi, $\mathrm{n}^{\circ}$ 11, pp. 27-36.

Lima L., Moulin S. (2006), « Justice et justifications dans les politiques de l'emploi », Travail et Emploi, $\mathrm{n}^{\circ} 105$, p. 55-67.

LomBa C. (2018), La Restructuration permanente de la condition ouvrière. De Cockerill à ArcelorMittal, Vulaines-sur-Seine, Éditions du Croquant.

Louey S., SchütZ G. (2014), «Les effets de la mixité au prisme du corps et de la sexualité : les hommes dans les métiers d'accueil », Travail et Emploi, n 140, pp. 5-19.

MARChal E. (1999), « Les compétences du recruteur dans l'exercice du jugement des candidats », Travail et Emploi, ${ }^{\circ}$ 78, pp. 41-51.

Maruani M., Nicole-Drancourt C. (1989), Au labeur des dames. Métiers masculins, emplois féminins, Paris, Syros.

Mauroux A. (coord.) (2016), « Chiffres clés sur les conditions de travail et la santé au travail », Synthèse. Stat', n ${ }^{\circ} 22$, Dares.

Méda D., Vendramin P. (2013), Réinventer le travail, Paris, Presses universitaires de France. 
Menoux T. (2016), Les Concierges d'hôtels : investissement dans un travail de service de luxe et construction collective du prestige d'un groupe professionnel, thèse de doctorat en sociologie, Paris, EHESS.

MetTe C. (2018), «En quoi les conditions de travail sont-elles liées au parcours professionnel antérieur? », Dares analyses, $\mathrm{n}^{\circ} 002$.

Mischi J. (2016), Le Bourg et l'atelier. Sociologie du combat syndical, Marseille, Agone.

MoliniÉ A.-F. (2003), « Interroger les salariés sur leur passé professionnel : le sens des discordances », Revue d'épidémiologie et de santé publique, vol. 51, n ${ }^{\circ}$ 6, pp. 589-605.

Molinié A.-F., LeroYer A. (2011), « Suivre les évolutions du travail et de la santé : EVREST, un dispositif commun pour des usages diversifiés », Perspectives interdisciplinaires sur le travail et la santé [en ligne], $\mathrm{n}^{\circ}$ 13-2. https://doi.org/10.4000/pistes.1852.

MoliniÉ A.-F., Volkoff S. (2016), « La construction et l'usage d'outils quantitatifs pour comprendre les conditions de travail », in Mahlaoui S., L'Articulation des démarches quantitative et qualitative pour analyser le travail : mythe ou réalité ?, Relief, $\mathrm{n}^{\circ}$ 52, Céreq, pp. 65-76.

MoUlÉVRIER P. (2010), « Les "banquiers solidaires" ou la légitimation d'une "profession économique" », Formation emploi, $\mathrm{n}^{\circ}$ 111, pp. 51-65.

Paillet A., Serre D. (2014), « Les rouages du genre. La différenciation des pratiques de travail chez les juges des enfants », Sociologie du travail, vol. 56, n 3, pp. 342-364.

PASSERON J.-C. (1991), Le Raisonnement sociologique. L'espace non-popperien du raisonnement naturel, Paris, Nathan.

PenefF J. (1997), « Le travail du chirurgien : les opérations à cœur ouvert », Sociologie du travail, vol. 39, n 3, pp. 265-296.

PenefF J. (2000), Les Malades des urgences. Une forme de consommation médicale, Paris, Métailié.

Pialoux M. (1995), «L'ouvrière et le chef d'équipe ou comment parler du travail ? », Travail et Emploi, $\mathrm{n}^{\circ}$ 62, pp. 4-39.

Pinto V., CARTron D., Burnod G. (2000), «Étudiants en fast-food : les usages sociaux d'un "petit boulot" », Travail et Emploi, n ${ }^{\circ} 83$, pp. 137-156.

PuEyo V. (2000), « La traque des dérives : expérience et maîtrise du temps, les atouts des “anciens" dans une tâche d'autocontrôle », Travail et Emploi, n " 84, pp. 63-73.

RENAHY N. (2010), Les Gars du coin. Enquête sur une jeunesse rurale, Paris, La Découverte.

Rouxel C., Virely B. (2012), « Les transformations des parcours d'emploi et de travail au fil des générations », in Insee, Emploi et Salaires. Édition 2012, Paris, Insee, coll. « Insee références », pp. 39-50.

SCHEPENS F., Zolesio E. (coord.) (2015), « La fabrication du soignant au travail », Travail et Emploi, $\mathrm{n}^{\circ} 141$.

Schwartz O. (1993), «L'empirisme irréductible », postface à Anderson N., Le Hobo, sociologie du sans-abri, Paris, Nathan, pp. 265-308. 
SERre D. (2009), Les Coulisses de l'État social. Enquête sur les signalements d'enfant en danger, Paris, Raisons d'agir.

SERRE D. (2012), « Travail social et rapport aux familles : les effets combinés et non convergents du genre et de la classe », Nouvelles Questions féministes, vol. 31, n 2 2, pp. 49-64.

Simonet M. (2018), Travail gratuit, la nouvelle exploitation?, Paris, Textuel.

STEVENS H. (2013), «Entre émancipation symbolique et reproduction sociale. Ethnographie d'une formation de "développement personnel” », Travail et Emploi, n 133, pp. 39-51.

Teiger C., Laville A. (1991), «L'apprentissage de l'analyse ergonomique du travail, outil d'une formation pour l'action », Travail et Emploi, n 47, pp. 53-62.

ThÉBAud-Mony A. (1992), « La connaissance des maladies professionnelles », Travail et Emploi, $\mathrm{n}^{\circ} 54$, pp. 87-99.

Thébaud-Mony A., Daubas-Letourneux V. (2001), « Les angles morts de la connaissance des accidents du travail », Travail et Emploi, n ${ }^{\circ}$ 88, pp. 25-42.

TRAVAIL ET EMPLOI (2012), dossier « Les risques psychosociaux au travail : d'une "question de société" à des questions scientifiques », n¹29.

TRÉmEAu C. (2017), S'informer, s'indigner, réclamer, revendiquer ou non en entreprise. Les jeunes salariés à l'épreuve de leurs droits, thèse de doctorat en sociologie, université de Nantes.

VÉzINA N., STOCK S. (2005), « Collaboration interdisciplinaire dans le cas d'une intervention ergonomique », in Volkoff S. (coord.), L'Ergonomie et les chiffres de la santé au travail : ressources, tensions et pièges, Toulouse, Octarès, pp. 87-108.

Villette M., Vuillermot C. (2005), Portrait de l'homme d'affaires en prédateur, Paris, La Découverte.

Volkoff S. (2008), « L'intensification du travail "disperse" les problèmes de santé », in Terssac G. (de), Saint-Martin C., Thébault C. (coord.), La Précarité : une relation entre travail, organisation et santé, Toulouse, Octarès, pp. 29-42.

Volkoff S., Molinié A.-F. (1982), «Quantifier les conditions de travail ? L'exemple de l'enquête nationale d'octobre 1978 », Travail et Emploi, nº 11, pp. 63-70.

WAINSZTOK H. (2017), « Le genre encadré. Ethnographie d'une formation au leadership pour aspirant·e.s cadres », Travail et Emploi, $\mathrm{n}^{\mathrm{o}} 151$, pp. 53-73.

WAltisPerger D. (2008), « Pénibilité du travail et sortie précoce de l'emploi », Premières Synthèses, nº3.1, Dares.

WEBER F. ([1989] 2009), Le Travail à-côté. Une ethnographie des perceptions, nouvelle éd. revue et augmentée, Paris, Éditions de l'École des hautes études en sciences sociales.

Wolff L., Mardon C., Molinié A.-F., Volkoff S., Gaudart C. (2015), « Les changements de conditions de travail au fil des vies professionnelles : plus fréquents, moins favorables », Connaissance de l'emploi, $\mathrm{n}^{\circ} 124$, CEE.

ZELIZER V. (2008), « L'Économie du care », Revue française de socio-économie, nº 2, pp. 13-25. 